\title{
Communicating results through crystallographic databases
}

Suzanna C. Ward ${ }^{1}$, Amy A. Sarjeant ${ }^{2}$, Matthew P. Lightfoot ${ }^{1}$

${ }^{1}$ The Cambridge Crystallographic Data Centre, 12 Union Road, Cambridge, CB2 1EZ, UK.

${ }^{2}$ The Cambridge Crystallographic Data Centre, 174 Frelinghuysen Rd. Piscataway, NJ 08854, USA.

E-mail: ward@ccdc.cam.ac.uk

Historically the only route to disseminate data including crystal structures was to publish the data through a scientific article. In the world of crystallography this data would then be abstracted after publication into crystallographic databases such as the Cambridge Structural Database (CSD).

Over the last decade, the landscape of data publishing has started to change. Firstly, crystallographers are no longer listed as an author by default on publications associated with the data they collect. This means that not only are they not being acknowledged for the work they have done but they may not be actively engaged in the peer review process. Secondly, and perhaps in some ways in response to this, we are seeing the rise of data journals and of data solely being published through databases. As a case in point, there were more structures published directly through the CSD as a CSD Communication in 2016 than in any other year and it became the most popular place to publish crystal structures. Other databases also provide this option and it can go some way to ensure that crystallographers get credit for their data.

This presentation will explore how crystallographic results are currently published through the scientific literature, data journals and databases. We will look at what the advantages and disadvantages of the different options might be and the role databases can play to help the crystallographic community adopt standards with respect to quality and ethics. The presentation will conclude by looking at how communicating crystallographic results might change in the future and how databases could help the community to share their structures and increase the credit they receive for their data. 